\title{
Impact of enhanced recovery after surgery or fast track surgery pathways in minimally invasive radical prostatectomy: a systematic review and meta-analysis
}

\author{
Zhengtong $\mathrm{Lv}^{1,2}$, Yi Cai ${ }^{1,2}$, Huichuan Jiang ${ }^{1,2}$, Changzhao Yang ${ }^{1,2}$, Congyi Tang ${ }^{1,2}$, Haozhe Xu ${ }^{1,2}, \mathrm{Zhi}^{1,2}$, \\ Benyi Fan ${ }^{1,2 \#}$, Yuan $\mathrm{Li}^{1,2 \#}$ \\ ${ }^{1}$ Department of Urology, ${ }^{2}$ National Clinical Research Center for Geriatric Disorders, Xiangya Hospital, Central South University, Changsha, China \\ Contributions: (I) Conception and design: Z Lv; (II) Administrative support: B Fan, Y Li; (III) Provision of study materials or patients: Y Cai, H Jiang; \\ (IV) Collection and assembly of data: C Yang, C Tang, H Xu, Z Li; (V) Data analysis and interpretation: Z Lv; (VI) Manuscript writing: All authors; \\ (VII) Final approval of manuscript: All authors. \\ \#These authors contributed equally to this work. \\ Correspondence to: Yuan Li; Benyi Fan. Department of Urology, Xiangya Hospital, Central South University, 87 Xiangya Rd., Changsha 410008, \\ China. Email: yuanlixy@csu.edu.cn; fanbenyi2009@yeah.net.
}

\begin{abstract}
Background: The enhanced recovery after surgery (ERAS) and fast track surgery (FTS) protocols have been applied to a variety of surgeries and have been proven to reduce complications, accelerate rehabilitation, and reduce medical costs. However, the effectiveness of these protocols in minimally invasive radical prostatectomy (miRP) is still unclear. Thus, this study aimed to evaluate the impact of ERAS and FTS protocols in miRP.
\end{abstract}

Methods: We searched PubMed, Cochrane Library, Embase, and Web of Science databases to collect randomized and observational studies comparing ERAS/FTS versus conventional care in miRP up to July 1, 2019. After screening for inclusion, data extraction, and quality assessment by two independent reviewers, the meta-analysis was performed with the RevMan 5.3 and STATA 15.1 software. Results were expressed as risk ratio (RR) and weighted mean difference (WMD) with $95 \%$ confidence intervals (CIs).

Results: In total, 11 studies involving 1,207 patients were included. Pooled data showed that ERAS/FTS was associated with a significant reduction in length of stay (LOS) (WMD: -2.41 days, 95\% CI: -4.00 to -0.82 days, $\mathrm{P}=0.003$ ), time to first anus exhaust (WMD: -0.74 days, $95 \% \mathrm{CI}:-1.14$ to -0.34 days, $\mathrm{P}=0.0003$ ), and lower incidence of postoperative complications (RR: 0.70, 95\% CI: 0.53 to $0.92, \mathrm{P}=0.01$ ). No significant differences were found between groups for operation time, estimated blood loss, postoperative pain, blood transfusion rate, and readmission rate $(\mathrm{P}>0.01)$.

Conclusions: Our meta-analysis suggests that the ERAS/FTS protocol is safe and effective in miRP. However, more extensive, long-term, prospective, multicenter follow-up studies, and randomized controlled trials (RCTs) are required to validate our findings.

Keywords: Enhanced recovery after surgery (ERAS); fast track; meta-analysis; prostate cancer; radical prostatectomy (RP)

Submitted Dec 18, 2019. Accepted for publication May 25, 2020.

doi: $10.21037 /$ tau-19-884

View this article at: http://dx.doi.org/10.21037/tau-19-884

\section{Introduction}

Prostate cancer is already the fourth most common cancer in the world and the second most common among men, and has become a significant threat to the health of older men (1). Despite the diversity of treatments for prostate cancer, radical prostatectomy $(\mathrm{RP})$ is still the most crucial treatment 
for low- and medium-risk prostate cancer (2). The increase in prostate-specific antigen (PSA) screening and improved prostate biopsy techniques have contributed to an increase of early-stage prostate cancers, which in turn has led to an increase in the number of RP candidates.

Over the past decade, laparoscopy and robotics have advanced leaps and bounds. The conventional open radical retropubic prostatectomy (RRP) has been almost replaced by minimally invasive radical prostatectomy (miRP) surgery. High-quality randomized controlled trials (RCTs) and subsequent meta-analysis have proven that miRP surgery can significantly improve surgical quality and reduce surgical complications $(3,4)$. However, postoperative complications and readmission still occur due to the factors of advanced age, improper care, and rehabilitation measures (5). Therefore, any ideas to reduce complications and promote recovery are worth investigating.

A second approach to promoting rehabilitation that has become popular in recent years is the enhanced recovery after surgery (ERAS) pathway, which is also known as fast track surgery (FTS). This multimodal treatment modality was initially reported in the 1990s by Kehlet et al. $(6,7)$; it aims to attenuate surgical stress, accelerate postoperative recovery, and shorten hospital length of stay (LOS) through a series of perioperative surgical, anesthetic, and nursing measures. Since the concept was first proposed, ERAS/ FTS pathways have been popularized worldwide and have revolutionized traditional thinking and behavior over the past 30 years. It has been applied in many surgical procedures attaining encouraging results, especially in digestive system surgery (8).

Currently, the field of urology surgery is endeavoring to use ERAS/FTS pathways in its practice. Many isolated RCTs and controlled clinical trials (CCTs) have tried to address the feasibility and superiority of ERAS/FTS for miRP surgery, yet the outcomes of these trials were inconsistent. For example, some studies have concluded that ERAS/FTS pathways can reduce the LOS (9-14), while other studies have contradicted this (15-17). Some research has shown that ERAS/FTS pathways can lower the rates of complications $(9,13)$, but other research did not bear this out $(10,12,14-16)$. Some studies have shown that ERAS/FTS pathways could shorten the time of catheter removal $(12,15,17)$, while others did not corroborate these findings $(14,16,18)$.

Given the striking variation of the related research, we conducted this review and meta-analysis for the first time to evaluate the effects of ERAS/FTS pathways in prostate cancer patients undergoing miRP surgery. We present the following article in accordance with the PRISMA reporting checklist (available at http://dx.doi.org/10.21037/tau-19884).

\section{Methods}

\section{Study aim}

This study aimed to explore whether the ERAS/FTS pathways can improve intraoperative and postoperative outcomes of miRP compared with the conventional or nonERAS/FTS mode. We followed the Preferred Reporting Items for Systematic Reviews and Meta-Analyses (PRISMA) statement. This meta-analysis is registered at PROSPERO, and the registration number is CRD42019138480.

\section{Literature search}

A systematic search of the databases of PubMed, Cochrane Library, Embase, and Web of Science databases up to July 1, 2019, was conducted to identify relevant studies without language restriction. We searched for the following medical subject heading terms and free-text terms individually or in combination: "Enhanced Recovery" OR "Early Recovery" OR "ERAS" OR "Fast track" OR "Accelerated rehabilitation" OR "Collaborative care pathway" OR "Clinical pathway" OR "Multimodal" AND "Prostatectomy" OR "Prostatectomies". The search strategies for each database are available in Table 1. Reference lists of all eligible articles were also searched for any other related studies. We also performed a manual search for abstracts from international meetings through Internet search engines, which were then downloaded and studied.

\section{Inclusion and exclusion criteria}

The inclusion criteria for this study were the following: (I) participants: patients who were diagnosed with prostate cancer, and underwent miRP, including laparoscopic radical prostatectomy (LRP) and robot-assisted radical prostatectomy (RARP); (II) intervention: ERAS/FTS protocol was proposed in the study, and if not, an ERAS/ FTS-like perioperative management protocol was used; (III) controls: the conventional protocol was applied for patients who underwent RP; (IV) outcomes: at least 1 of the main outcomes of interest.

The exclusion criteria were the following: (I) the 
Table 1 Search strategies for each database

\begin{tabular}{|c|c|}
\hline Search number & Queries \\
\hline$\# 1$ & Search "Prostatectomy"[Mesh] \\
\hline \#2 & Search ((Prostatectomies[Title/Abstract]) OR Prostatectomy[Title/Abstract]) \\
\hline \#3 & \#1 OR \#2 \\
\hline \#5 & \#3 AND \#4 \\
\hline \multicolumn{2}{|c|}{ Queries in Cochrane } \\
\hline$\# 1$ & MeSH descriptor: [Prostatectomy] explode all trees \\
\hline \#4 & $\begin{array}{l}\text { (Enhanced Recovery):ti,ab,kw OR (ERAS):ti,ab,kw OR (Fast track):ti,ab,kw OR (Accelerated rehabilitation):ti,ab,kw } \\
\text { OR (Collaborative care pathway):ti,ab,kw OR (Clinical pathway):ti,ab,kw OR (Multimodal):ti,ab,kw }\end{array}$ \\
\hline \#5 & \#3 AND \#4 \\
\hline \multicolumn{2}{|l|}{ Queries in WOS } \\
\hline$\# 1$ & TS=(Prostatectomy OR Prostatectomies) \\
\hline \#2 & $\begin{array}{l}\mathrm{TI}=(\text { Enhanced Recovery OR Early recovery OR ERAS OR Fast track OR Accelerated rehabilitation OR Collaborative } \\
\text { care pathway OR Clinical pathway OR Multimodal) }\end{array}$ \\
\hline \#3 & \#1 AND \#2 \\
\hline
\end{tabular}

inclusion criteria were not met; (II) the outcomes of interest were not reported or impossible to calculate or extrapolate; (III) studies were reviews, meta-analyses, case reports, letters, or protocols; (IV) repeated publication of the same study population.

\section{Study selection and data extraction}

Two researchers ( $\mathrm{Y}$ Cai and $\mathrm{H}$ Jiang) identified and selected citations from the databases independently. Any disagreements between the two were settled by a deciding arbiter (B Fan). Similarly, data were independently extracted from all the included articles by two authors ( $\mathrm{H} \mathrm{Xu}$ and $\mathrm{Z} \mathrm{Li}$ ). Any discrepancies or divergences concerning the availability of data were settled through discussion with a third reviewer (Y Li). The following information was extracted: first author, year of publication, country, study design, matching factors, intervention items, and outcomes of interest.

\section{Qualitative evaluation of cited studies}

Two independent reviewers (C Yang and C Tang) separately assessed the quality of cited studies, and if there was a disagreement, Y Li would be the arbiter to resolve differences. The quality of identified RCTs was assessed with the Cochrane risk of bias tool, which included selection, performance, detection, attrition, reporting, and other biases. The risk bias of non-randomized studies was 
assessed by the Newcastle-Ottawa Scale, which included adequacy selection of cohort, comparability of studies, and outcome assessment.

\section{Outcomes}

The outcome measures were LOS, operation time, estimated blood loss, complications, time to first anus exhaust, postoperative pain score, blood transfusion rate, and readmission rate.

\section{Statistical analysis}

All statistical analyses were conducted using RevMan 5.3 and STATA 15.1 software. Since median and range or interquartile range cannot be included in the final statistical analysis, we converted them into mean and standard deviation using methods described by Wan et al. (19) and Luo et al. (20). The weighted mean differences (WMDs) and risk ratios (RRs) were respectively used to compare continuous and dichotomous variables. All results were reported with $95 \%$ confidence intervals (CIs). Heterogeneity was assessed using $\mathrm{I}^{2}$ and chi-squared tests and judged to be of substantial heterogeneity if the $\mathrm{I}^{2}$ value was $>50 \%$ and according to a $\mathrm{P}$ value of $<0.10$; a randomeffects model was then used. Otherwise, a fixed-effects model was used. To further test the stability of pooled estimates, a sensitivity analysis was performed by removing individual studies sequentially to detect the impact of each study on the overall estimates. Publication bias was estimated by the use of funnel plots and Egger's test.

\section{Results}

\section{Characteristics and quality of eligible studies}

A total of 1,744 studies were initially identified through the above search strategies, and 12 articles were selected through inclusion and exclusion criteria. However, only 11 studies were included in the final meta-analysis because 1 study involved both open and minimally invasive approaches, making it impossible to extract the data of minimally invasive surgery alone. These 11 studies included 2 RCTs $(9,18)$ and 9 CCTs $(10-17,21)$ (Figure 1). These studies involved a total of 1,207 patients, of which 579 received ERAS/FTS protocol and 628 received conventional or non-ERAS/FTS protocol. Table 2 summarizes the basic characteristics of the included studies, and Table 3 provides a detailed summary of all the included patients' clinical baseline information. The baseline characteristics were homogeneous between the two groups; 7 of the 9 CCTs scored 7 points or more using the Newcastle-Ottawa scale, and 2 were not assessed due to the limited information in the conference abstract (Table 4). Both RCTs had a substantial risk of performance and detection bias (Table 5) because the participants and evaluators could not be blinded due to different protocols.

\section{Outcome measures}

\section{LOS}

A total of nine studies reported LOS involving 1,066 participants. The forest plot showed that the ERAS/FTS group could significantly shorten LOS compared to the conventional group (WMD: -2.41 days, $95 \%$ CI: -4.00 to -0.82 days, $\mathrm{P}=0.003$, random effects model), with significant heterogeneity $\left(\mathrm{I}^{2}=98 \%, \mathrm{P}<0.00001\right)$ (Figure 2). The sensitivity analysis showed that the original analysis was not changed by omitting one study in each turn (Figure 3). There was no evidence of publication bias of LOS by either the funnel plot or Egger's test $(\mathrm{P}=0.693)$ (Figure 4).

\section{Operation time}

For operation time, seven studies reported operation time involving 790 participants. There was no significant difference in the operation time between the two groups (WMD: $-23.79 \mathrm{mins}, 95 \% \mathrm{CI}:-47.90$ to $0.32 \mathrm{mins}$, $\mathrm{P}=0.05$, random effects model), and there was significant heterogeneity $\left(\mathrm{I}^{2}=93 \%, \mathrm{P}<0.00001\right)$ (Figure 2). The sensitivity analysis showed that Parrado et al.'s study (15) had a significant impact on the overall results when it was removed (Figure 3). There was no significant evidence revealed by either the funnel plot or Egger's test $(\mathrm{P}=0.322)$ for publication bias (Figure 4).

\section{Estimated blood loss}

A total of six studies reported estimated blood loss involving 712 participants. The forest plot showed that ERAS/FTS group significantly reduced estimated blood loss compared to the conventional group (WMD: $-10.19 \mathrm{~mL}, 95 \% \mathrm{CI}$ : -18.19 to $-2.18 \mathrm{~mL}, \mathrm{P}=0.01$, fixed effects model), without significant heterogeneity $\left(\mathrm{I}^{2}=28 \%, \mathrm{P}=0.22\right)$ (Figure 2). The sensitivity analysis showed that Lin et al.'s study (14) had a significant impact on the overall results when it was removed (Figure 3). There was no significant evidence 


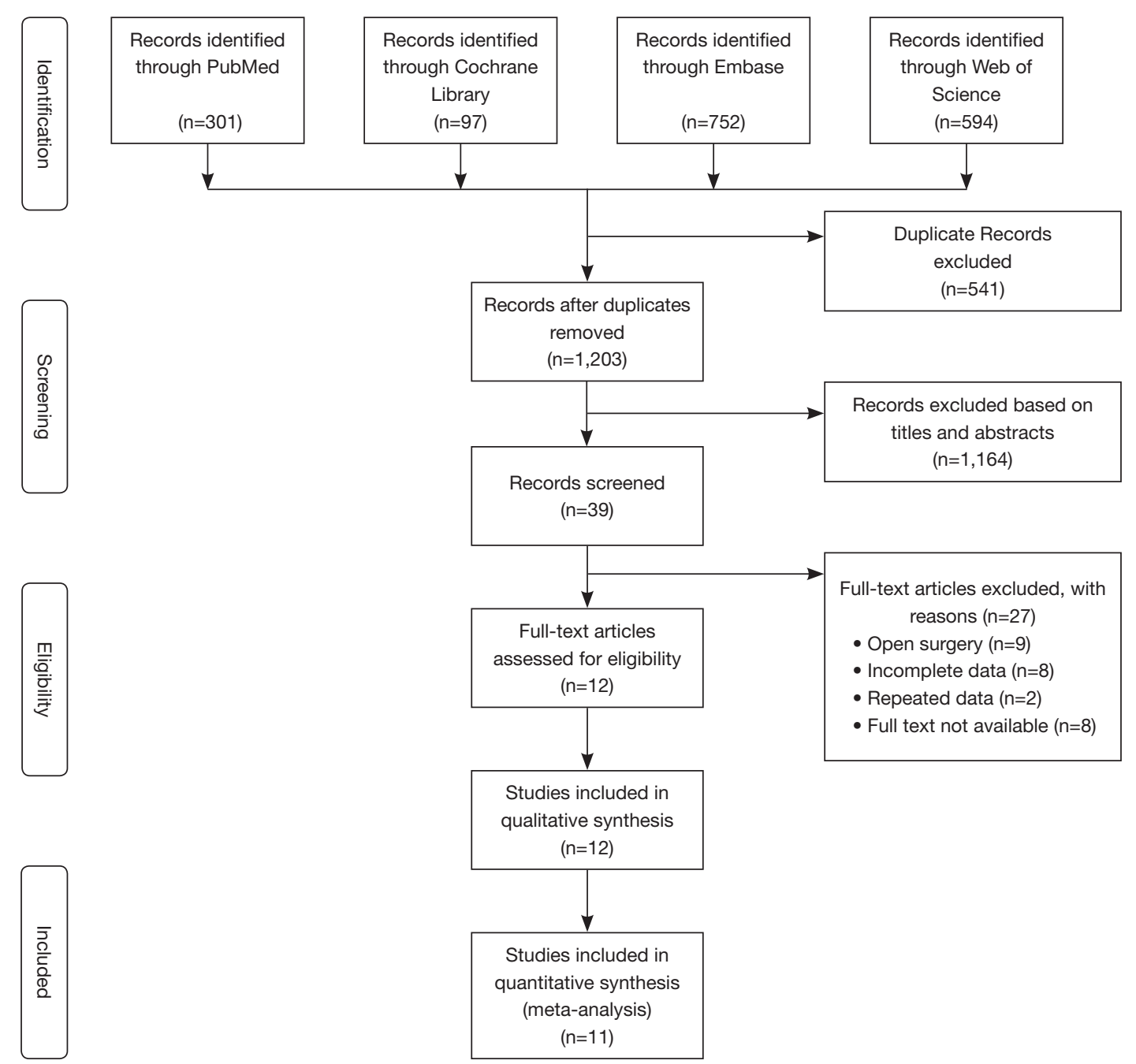

Figure 1 Preferred reporting items for systematic reviews and meta-analyses flow diagram.

found by either the funnel plot or Egger's test $(\mathrm{P}=0.198)$ for publication bias (Figure 4).

\section{Complications}

A total of seven studies reported complications involving 907 participants. The forest plot showed that the rate of overall complications was lower in the ERAS/FTS group (RR: $0.70,95 \%$ CI: 0.53 to $0.92, P=0.01$, fixed effects model), without significant heterogeneity $\left(\mathrm{I}^{2}=0 \%, \mathrm{P}=0.52\right)$ (Figure 2). The sensitivity analysis showed that the original analysis was not substantially changed by omitting one study in each turn (Figure 3). There was no significant evidence revealed by either the funnel plot or Egger's test $(\mathrm{P}=0.492)$ for publication bias (Figure 4).

\section{Other outcomes}

In other outcomes, the time to first anus exhaust was significantly shorter in the ERAS/FTS group compared with the conventional group (WMD: -0.74 days, $95 \%$ CI: -1.14 to -0.34 days, $\mathrm{P}=0.0003$, random effects model, $\mathrm{N}=3$ studies) (Figure 5A). There was no significant difference between the two groups in terms of postoperative pain score, blood transfusion rate, and readmission rate (WMD: -0.40 points, $95 \%$ CI: -1.51 to 0.70 points, $\mathrm{P}=0.47$, random effects model, $\mathrm{N}=2$ studies; RR: $0.42,95 \%$ CI: 0.06 to 3.16 , $\mathrm{P}=0.40$, random effects model, $\mathrm{N}=4$ studies; RR: $1.48,95 \%$ CI: 0.43 to $5.10, \mathrm{P}=0.54$, fixed effects model, $\mathrm{N}=3$ studies) (Figure 5B,C,D). Due to the limited number of studies, we did not conduct publication bias detection and sensitivity 
Table 2 Study characteristics

\begin{tabular}{|c|c|c|c|c|c|c|c|c|c|}
\hline Study & Year & Country & Design & \multicolumn{2}{|c|}{ No. of patients } & Approach & Interventions ${ }^{\dagger}$ & Outcomes reported $^{\ddagger}$ & Study quality \\
\hline Gralla et al. & 2007 & Germany & $\mathrm{RCT}$ & 25 & 25 & LRP & $\begin{array}{c}\mathrm{A}, \mathrm{C}, \mathrm{D}, \mathrm{F}, \mathrm{G}, \mathrm{H}, \\
\mathrm{I}, \mathrm{J}\end{array}$ & $1,2,3,4,12$ & $\begin{array}{c}\text { Moderate risk } \\
\text { of bias }\end{array}$ \\
\hline Parrado et al. & 2008 & Spain & ССT & 60 & 26 & LRP & ๆ & $1,2,4,5,6,7,9,11$ & High quality \\
\hline Hiba et al. & 2014 & Canada & CCT & 99 & 100 & $\begin{array}{l}\text { RRP, LRP, } \\
\text { RARP }\end{array}$ & $\underset{\mathrm{K}}{\mathrm{A}, \mathrm{B}, \mathrm{C}, \mathrm{H}, \mathrm{I}, \mathrm{J}}$ & $1,4,11,12$ & High quality \\
\hline Melinda et al. & 2016 & USA & СCT & 9 & 9 & RARP & $\mathrm{A}, \mathrm{E}, \mathrm{F}, \mathrm{H}, \mathrm{I}, \mathrm{J}$ & $2,3,13,14$ & High quality \\
\hline Yaiesh et al. & 2016 & Kuwait & CCT & 21 & 12 & RARP & ๆ & 1 & Unclear \\
\hline Huang et al. & 2018 & China & СCT & 36 & 37 & RARP & A, B, C, F, H, I, J & $1,2,3,4,7,8$ & High quality \\
\hline Graham et al. & 2019 & USA & CCT & 63 & 63 & RARP & ๆ & $1,5,10$ & Unclear \\
\hline Lin et al. & 2019 & China & СCT & 124 & 164 & LRP & $\begin{array}{c}A, B, C, D, E, F \\
\text { G, H, J, K }\end{array}$ & $1,2,3,4,5,6,7,8,9,12$ & High quality \\
\hline
\end{tabular}

\begin{abstract}
${ }^{\dagger}$, A: patient education; B: non-strict preoperative bowel preparation; C: non-strict preoperative fasting; D: intraoperative warming; E: optimization of anesthesia; F: goal-directed fluid management; G: reduce unnecessary indwelling of wound drainage tubes; $\mathrm{H}$ : Encourage early mobilization; I: encourage early oral feeding; J: enhanced postoperative analgesia; K: early removal of drainage tube or catheter; ${ }^{\ddagger}$, 1: LOS; 2: operation time; 3: estimated blood loss; 4: complications; 5: time of catheter removal; 6: time of drainage-tube removal; 7: time of taking food; 8: time of anus exhaust; 9: time of postoperative activity; 10: postoperative pain score; 11: transfusion; 12: readmission; 13: positive lymph nodes; 14: positive surgical margins; ${ }^{\S}$, the Cochrane risk of bias tool for RCTs and the Newcastle-Ottawa Scale for CCTs; ๆ, there are no detailed ERAS/FTS items in these three studies, but the authors explicitly proposed ERAS/FTS-like protocol in these studies. RCT, randomized controlled trial; CCT, controlled clinical trial; LRP, laparoscopic radical prostatectomy; RRP, radical retropubic prostatectomy; RARP, robot-assisted radical prostatectomy; LOS, length of stay; ERAS, enhanced recovery after surgery; FTS, fast track surgery.
\end{abstract}

analysis for the above results.

\section{Discussion}

ERAS/FTS is an innovative concept and a new rehabilitation model that in the recent years has been applied to the perioperative surgical period. It has disrupted the long-term concepts and principles of surgical therapeutics, and even overturned the traditional clinical medical understandings and technical norms with its superior social, scientific, and economic effects (22). After the ERAS Society was formed in 2010, it published a series of ERAS guidelines related to colorectal surgery, gastrointestinal surgery, gynecologic surgery, orthopedics surgery, cystectomy, etc. (http://www. erassociety.org). However, thus far, there has been no guideline or expert consensuses established in ERAS/FTS for RP. Although 2 articles have reported the possibility of discharge on the same day undergoing miRP, we do not have sufficient evidence to prove that this extreme model can be carried out globally due to the single-center, small sample size, medical conditions, and ethnic differences of these reports $(21,23)$. As is widely known, RP is the most difficult and complex operation in urology: it requires strict perioperative care and is completely constituted of patients who are older men. These factors all complicate the effectiveness and safety of ERAS/FTS. Therefore, we were motivated to complete this review and meta-analysis to evaluate the safety and effectiveness of ERAS/FTS in RP.

As the first recommended treatment for localized prostate cancer, RP surgery has gradually evolved from open surgery to LRP surgery and RARP surgery. The advent of minimally invasive surgery has dramatically 


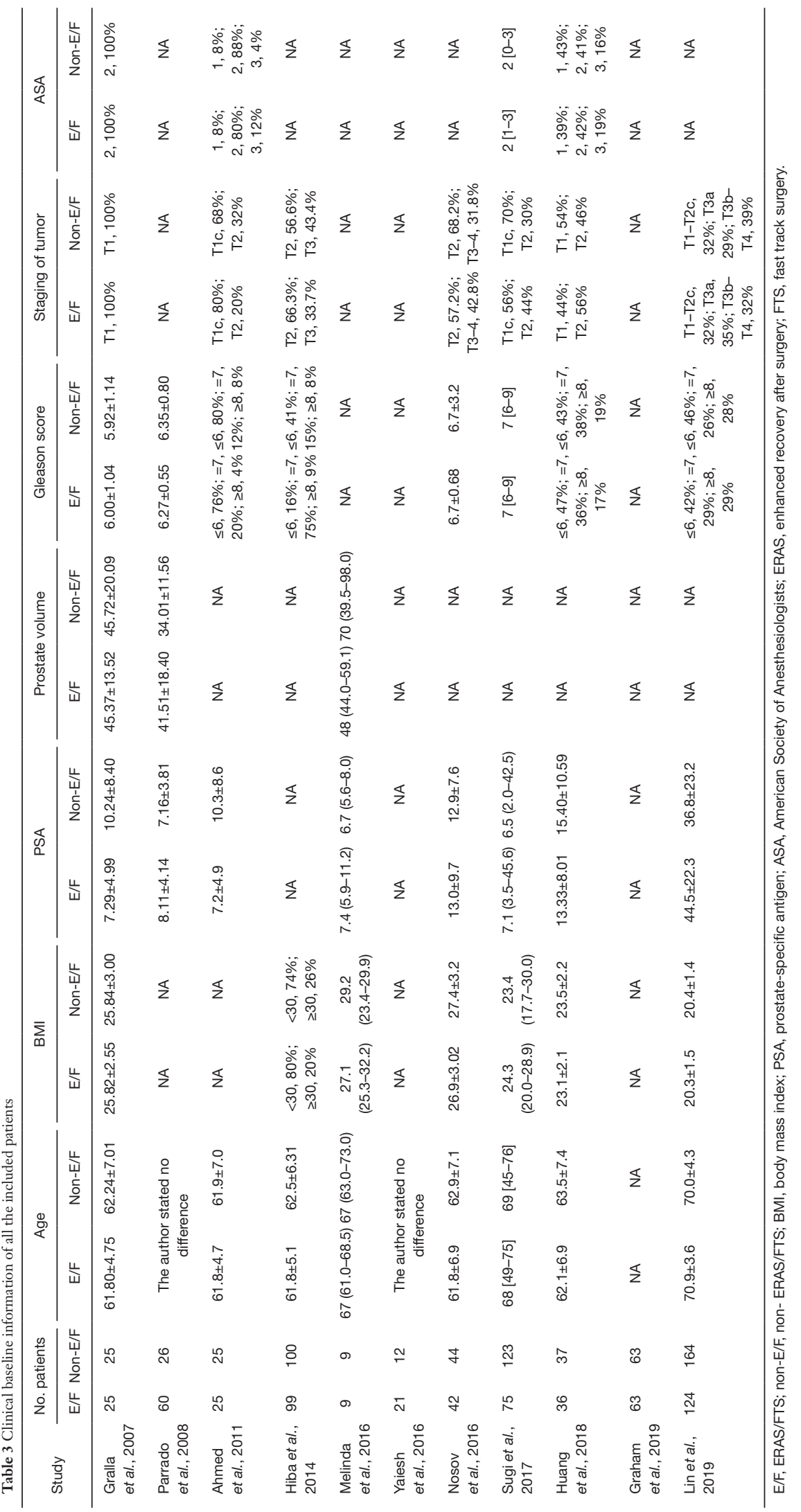




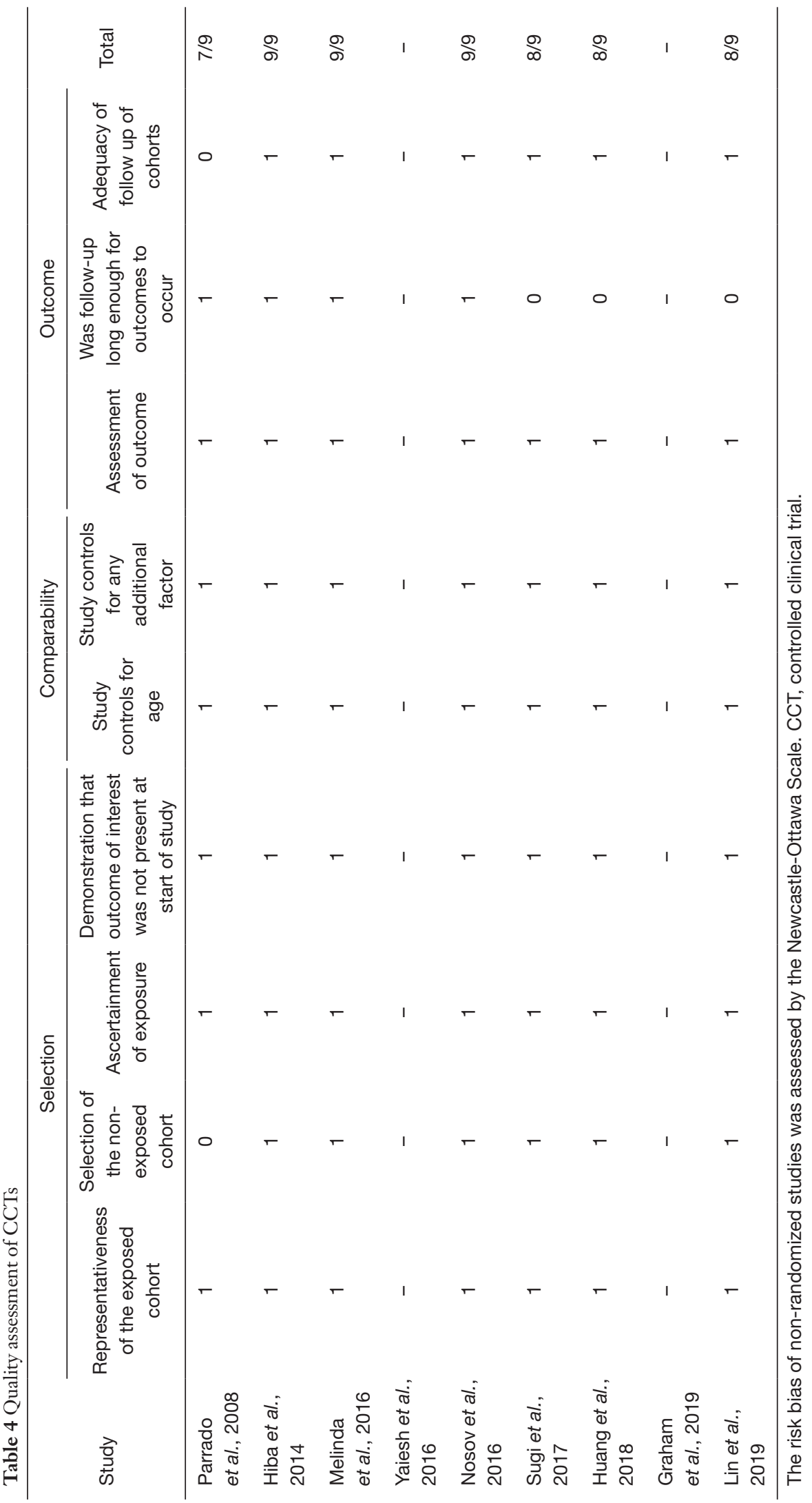


Table 5 Quality assessment of RCTs

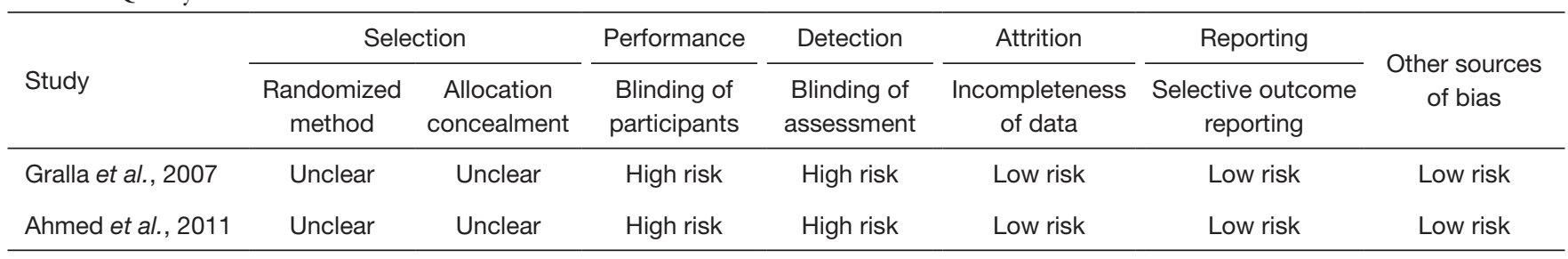

The quality of identified RCTs were assessed by the Cochrane risk of bias tool. RCT, randomized controlled trial.

reduced blood loss, transfusion rate, and LOS, and has thus gradually phased out open surgery (24). Therefore, to avoid the heterogeneity of results and provide more practical evidence for modern surgical medicine, this meta-analysis only included minimally invasive surgery.

Our findings showed that the ERAS/FTS could shorten LOS, time to first anus exhaust, and reduce the incidence of complications, while not aggravating the patient's postoperative pain. It is worth mentioning that the ERAS/FTS program also did not increase the risk of blood transfusion and readmission; the former has been associated with adverse survival and recurrence outcomes (25), while the latter may increase medical costs and indicate more serious post-discharge adverse events (26).

There are several pathophysiological reasons why ERAS/ FTS can achieve such encouraging clinical results. First, preoperative education and psychological counseling are necessary to introduce the detailed ERAS/FTS program, which can ease the psychological pressure and help patients better understand and coordinate the ERAS/FTS protocol. Second, the ERAS/FTS protocol eliminates the need for mechanical bowel preparation, which is associated with dehydration and changes in electrolyte balance (particularly in the elderly) (27). Third, the ERAS/FTS protocol reduces time of fasting and increases carbohydrate intake in the preoperative period, which reduces the prevalence of preoperative thirst, hunger, and anxiety; decreases the loss of nitrogen and protein after surgery; helps retain more lean body mass and muscle strength; and decreases the insulin resistance in the postoperative period (28). Fourth, goal-directed fluid management can reduce the incidence of intestinal obstruction by the maintenance of splanchnic perfusion and adequate systemic oxygenation (29). Fifth, the optimization of anesthesia, such as epidural anesthesia use, has shown beneficial effects on perioperative pulmonary function, blunting the surgical stress response, and improved analgesia (30). Sixth, intraoperative warming, early mobilization, and early postoperative oral feeding play a vital role in accelerating recovery for patients by maintaining body homeostasis as early as possible (28).

In addition, the ERAS/FTS model of RP has some of its own unique interventions. One is to reduce the number and the indwelling time of pelvic drainage tubes. It is well known that standard RP is often accompanied by pelvic lymph node dissection, which routinely requires pelvic drainage tubes to drain lymph and some bloody fluid. However, pelvic drainage not only brings inconvenience to patients' normal activities and affects post-operative rehabilitation, but is also associated with infection, pain, prolonged hospitalization, and increased costs $(31,32)$. Moreover, a case of pulmonary embolism was reported in a patient due to pelvic venous compression caused by drainage tubes after RP (33). Therefore, the use of pelvic drains is controversial in RP. Several studies have proven that routine drainage tube placement following RP did not confer a significant advantage in terms of postoperative complications (34-37). In our meta-analysis, three of the included studies described the reduced indwelling time of the pelvic drainage tube and also demonstrated that it was safe $(12,14,15)$.

Another specialist intervention for ERAS/FTS following $\mathrm{RP}$ is to shorten the indwelling time of the catheter. RP has historically been associated with prolonged catheterization to allow anastomotic healing in most urological centers. Early removal of the catheter may cause urine leakage, anastomotic strictures, and urinary incontinence, although it has not been proven (38). However, the long-term indwelling of catheters can lead to various catheter-related problems including urinary tract infections, traumatic injuries of the urethra, bleeding, obstruction, pain, discomfort, and bladder spasms, and all of these adverse effects exert a negative influence on health-related quality of life (39). Recently, several large, high-quality studies have confirmed that early removal of the catheter has no adverse impact on catheter-related complications (40-42), which is also consistent with the studies included in our meta- 


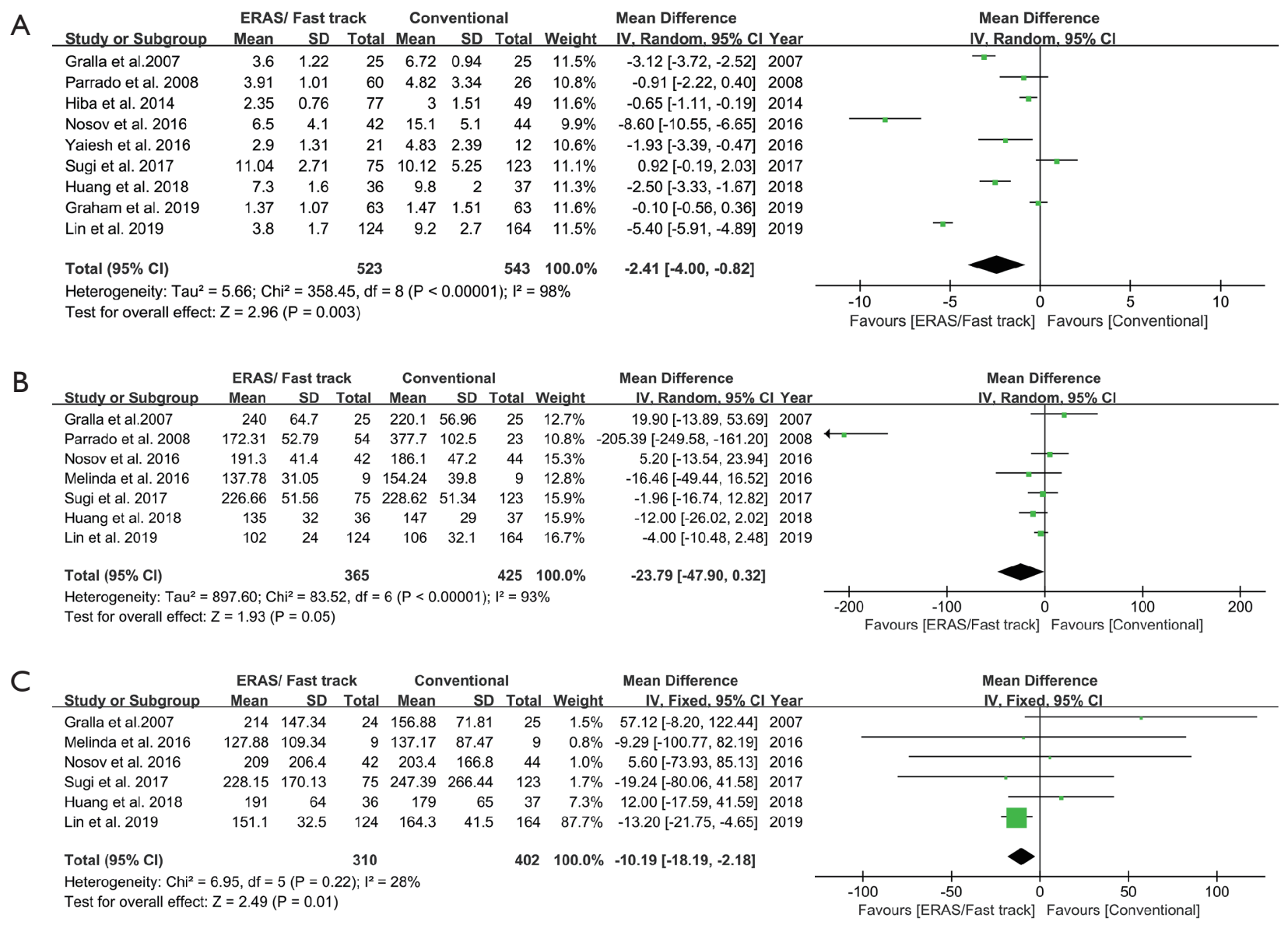

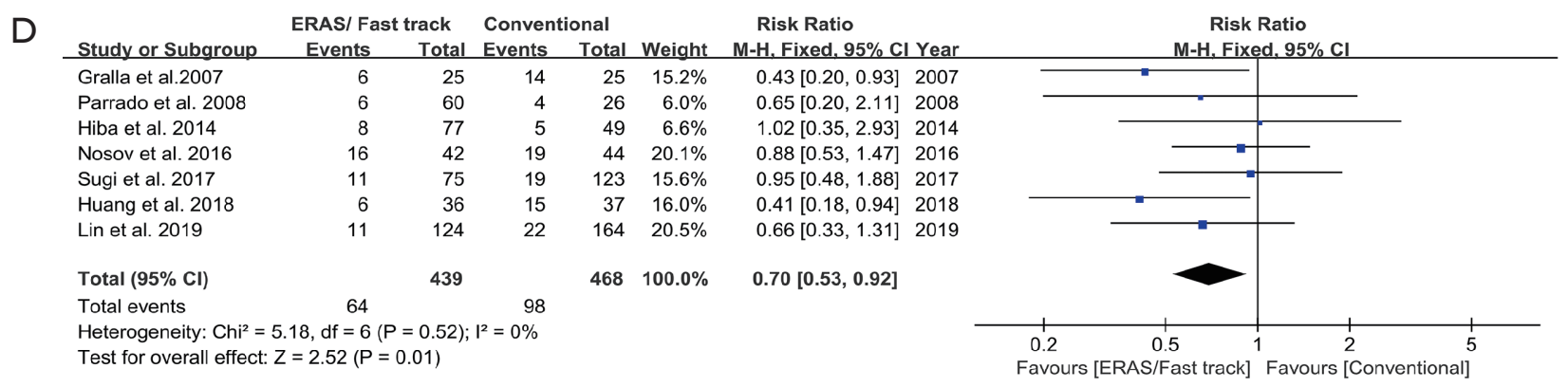

Figure 2 Forest plot evaluating the outcomes of (A) LOS, (B) operation time, (C) estimated blood loss, and (D) complications between ERAS/FTS and conventional care. The size of squares corresponds to the weight of the study in the meta-analysis. The diamond plot represents the overall results of the included trials. The horizontal lines represent the 95\% CI. LOS, length of stay; ERAS, enhanced recovery after surgery; FTS, fast track surgery; 95\% CI, 95\% confidence interval; df, degrees of freedom; IV, inverse variance test; M-H, Mantel-Haenszel test.

analysis (12,14-17).

Two controversial results need to be discussed specifically. The first is the result of the operation time, which seemed to be an illusion wherein ERAS shortened the operation time when all the data were aggregated
(WMD: -23.79 mins, 95\% CI: -47.90 to $0.32 \mathrm{mins}$, $\mathrm{P}=0.05)$. However, the sensitivity analysis showed that when Parrado et al.'s study was removed, there was no significant difference in the operation time between the ERAS/FTS and conventional groups (WMD: -3.89 mins, 95\% CI: 
A

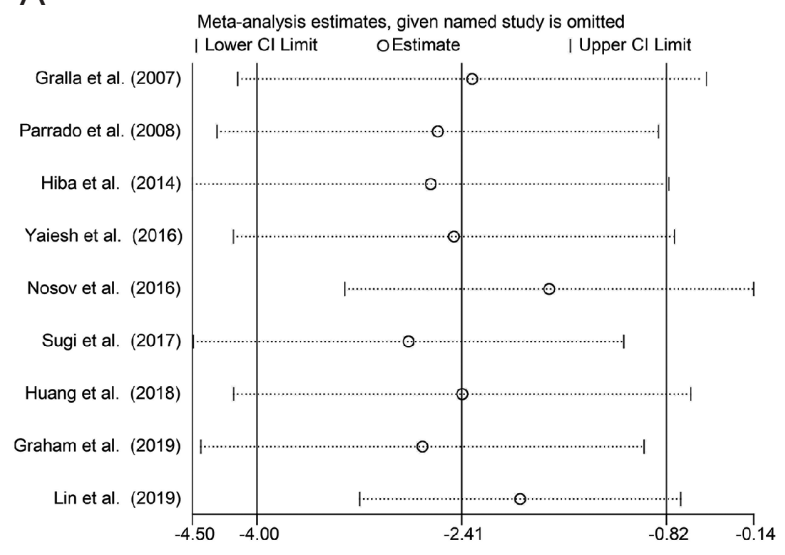

C

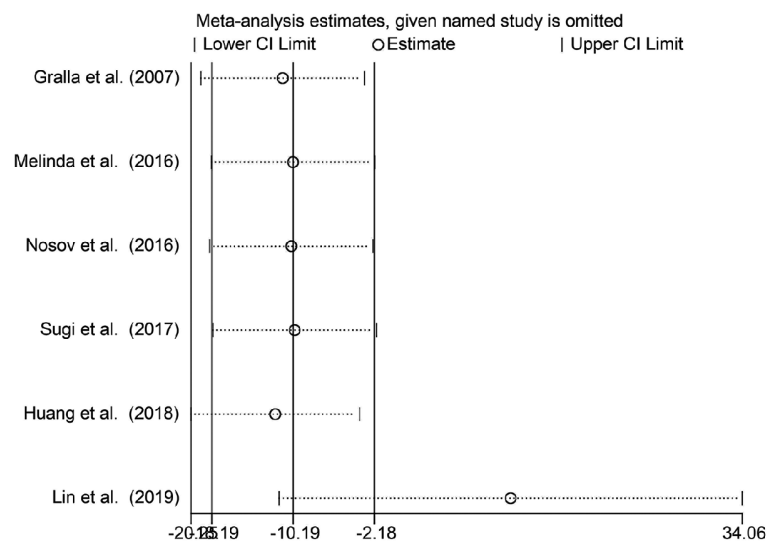

B

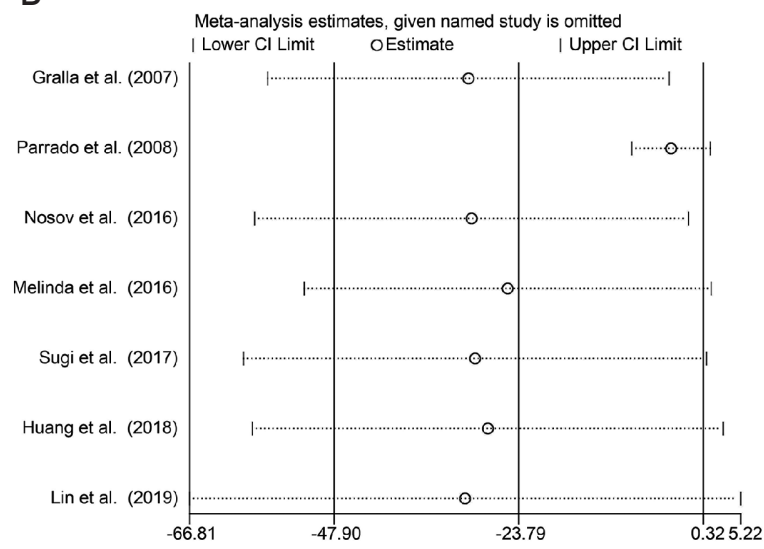

D

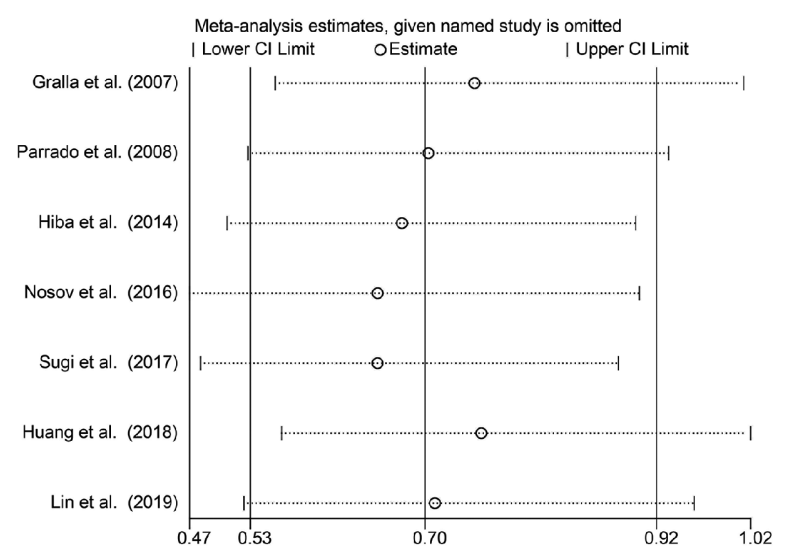

Figure 3 Sensitivity analysis of (A) LOS, (B) operation time, (C) estimated blood loss, and (D) complications. The 3 vertical lines represent the overall effect value and its $95 \%$ CI. Each circle and horizontal line represent the effect value and $95 \%$ CI after removing each indicated study. LOS, length of stay; $95 \%$ CI, 95\% confidence interval.

-9.01 to 1.23 mins, $\mathrm{P}=0.14)$. So, we reread this study and found that the control group underwent LRP in 2002, while the ERAS/FTS group received LRP from 2004 to 2005. The reason why the operation time was significantly reduced after the ERAS/FTS implementation $(172.31 \pm 52.79$ vs. $377.70 \pm 102.50$ mins, $\mathrm{P}<0.01$ ), as explained in detail by the author, was due to the greater experience acquired by the staff throughout time rather than ERAS interventions. The second one was the estimated blood loss, which also appeared to be significantly reduced in the ERAS/FTS group (WMD: $-10.19 \mathrm{~mL}, 95 \% \mathrm{CI}:-18.19$ to $-2.18 \mathrm{~mL}$, $\mathrm{P}=0.01$ ). However, the sensitivity analysis showed that when removing the study of Lin et al., we also found that ERAS/FTS group did not show any advantage in terms of estimated blood loss (WMD: $11.26 \mathrm{~mL}, 95 \% \mathrm{CI}:-11.54$ to
$36.06 \mathrm{~mL}, \mathrm{P}=0.33)$. We also reread this article and found that although less blood loss subjectively appeared in the ERAS/FTS group, there was no statistical difference related to this $(151.1 \pm 32.5$ vs. $164.3 \pm 41.5 \mathrm{~mL}, \mathrm{P}=0.143)$. When we looked back to the forest plot, we found the weight of this study was the highest of all the six studies included, reaching up to $87.7 \%$, which deeply affected the overall meta-result (Figure 2C). Therefore, we do not have sufficient evidence or any convincing explanation to prove that ERAS/FTS protocol can reduce intraoperative bleeding.

Although all the included studies were biased in favor of ERAS/FTS, we should be objective and rational to avoid the misinformation caused by an overemphasis on positive results. A study involving 1,301 patients found that more than half of the patients could not tolerate early 


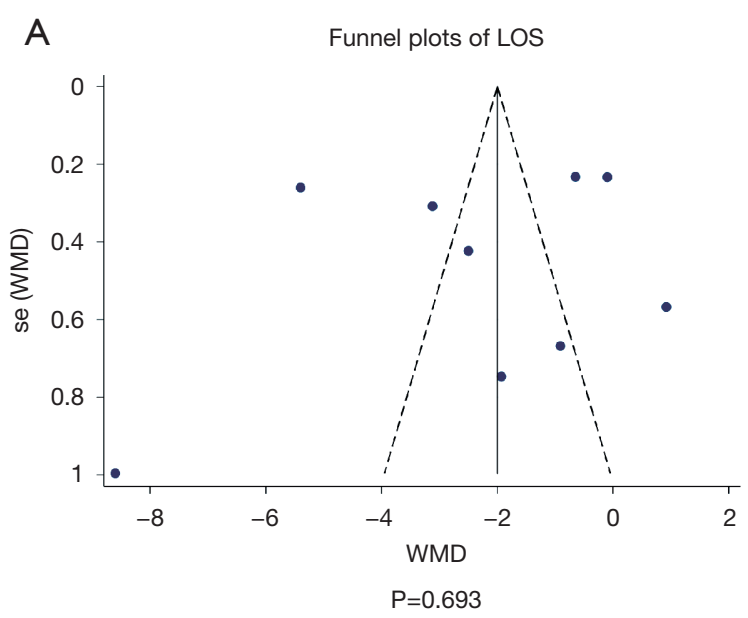

C

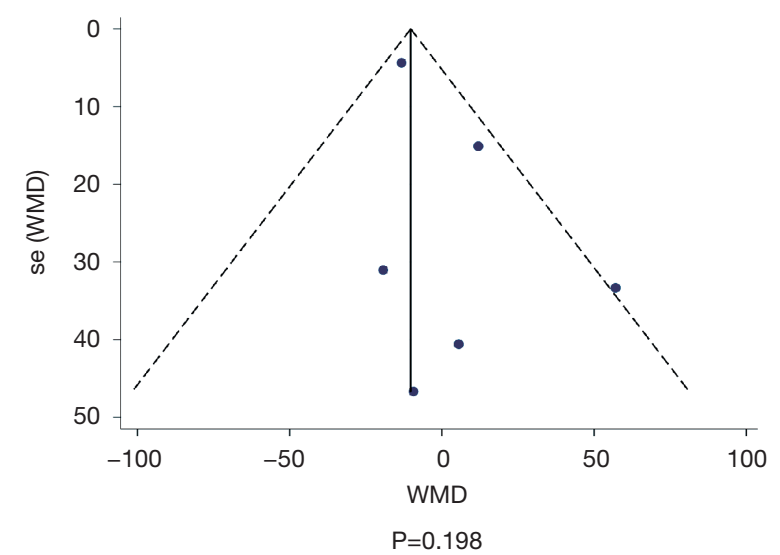

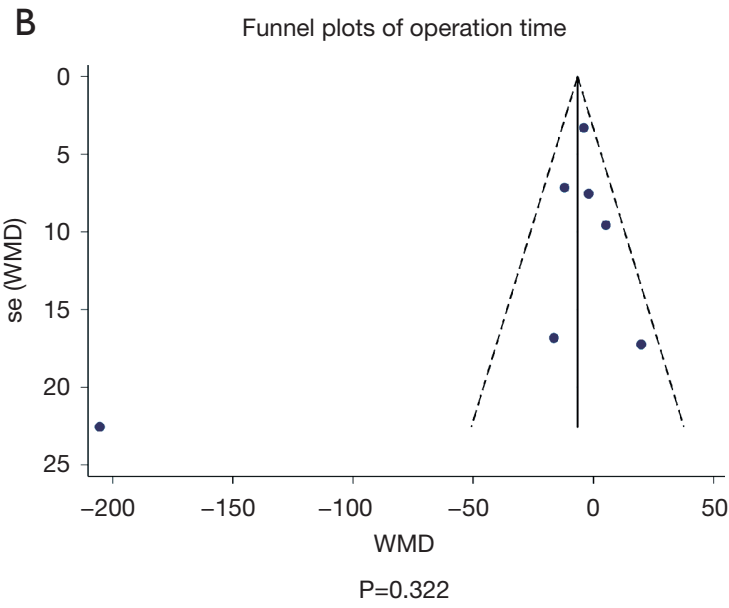

D Funnel plots of complications

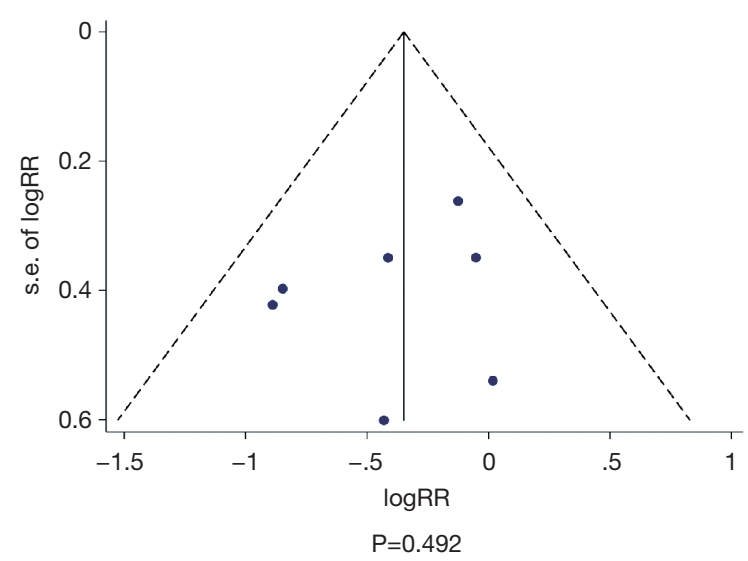

Figure 4 The funnel plots of (A) LOS, (B) operation time, (C) estimated blood loss, and (D) complications. The funnel plots seem symmetric, and Egger's test is non-significant, which suggests that there was no publication bias. LOS, length of stay.

enteral realization, and forced eating was associated with a higher risk of postoperative complications (43). We should consider adapted protocols with tailored procedurespecific re-nutrition policies, especially for patients with poor gastrointestinal function. Early mobilization is also considered essential in an ERAS/FTS program. However, swelling and discoloration of the scrotum in some patients after laparoscopic surgery may impair the patient's ability or willingness to mobilize early (9). Not only that, it is well recognized that some patients might experience repeated dizziness, nausea, or even fainting during early postoperative mobilization (44). When we encourage early mobilization, sufficient protective interventions must be in place; for example, a jockstrap for modest pressure on the scrotum can avoid fluid influx and reduce swelling in the scrotal and penile tissue (9). In conclusion, despite the global success of ERAS/FTS pathways, many challenges remain. Future optimizations should be made on a processspecific and patient-specific basis.

Despite our careful work on this meta-analysis of the currently available evidence, the findings should be interpreted in the context of known limitations. Firstly, the quantity and quality of the studies included were limited. Only two studies were RCTs and has a potentially substantial risk of bias, while the remaining studies were CCTs. Therefore, the outcomes of this study might have been influenced by information bias, selection bias, detection bias, and confounding bias. Secondly, although we excluded open surgery, it was undeniable that heterogeneity objectively existed between LRP and RARP. However, we did not conduct a subgroup analysis due to the limitation of the number of studies. Thirdly, the ERAS/FTS program 


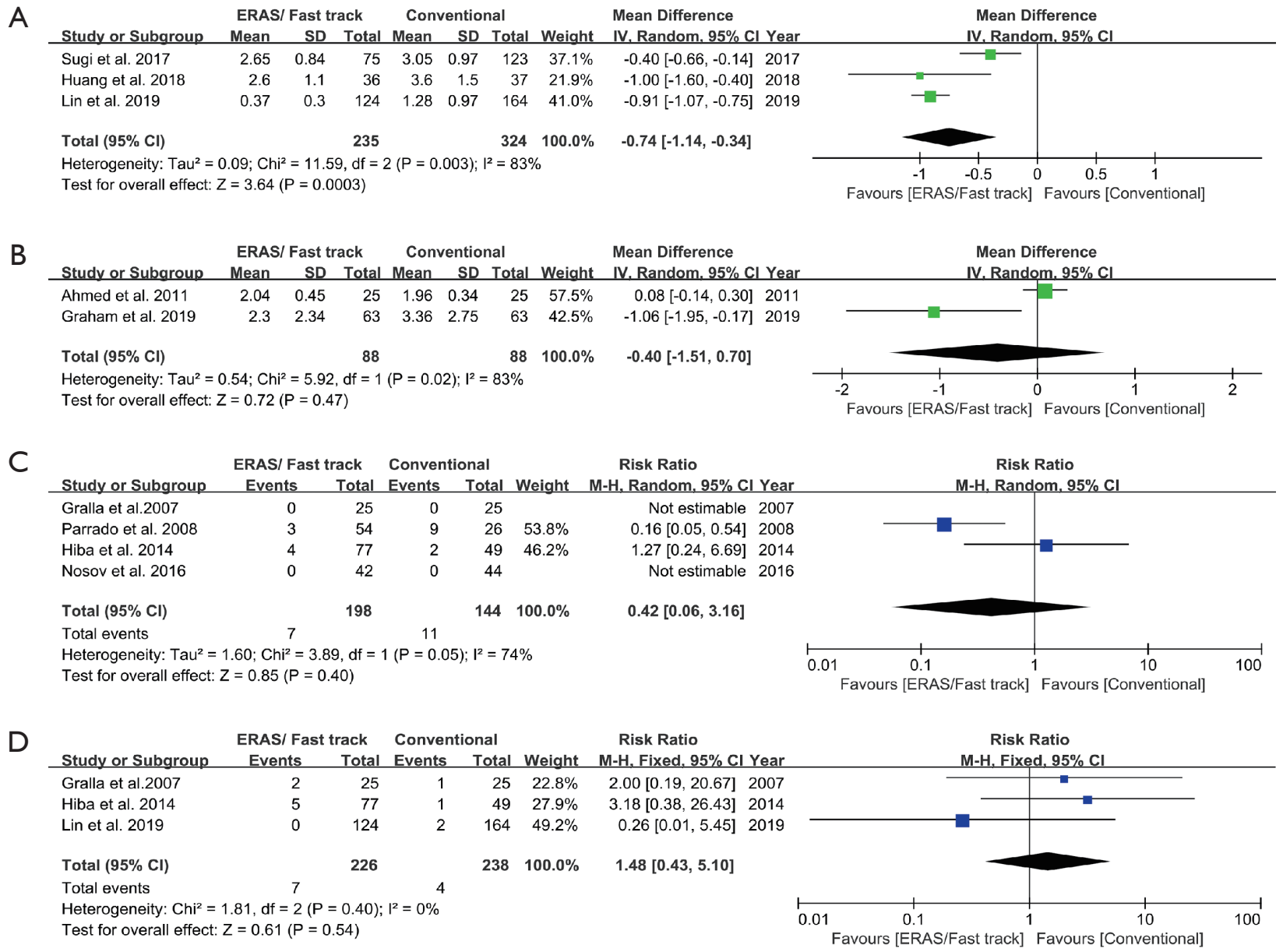

Figure 5 Forest plot evaluating the outcomes of (A) time to first anus exhaust, (B) postoperative pain score, (C) blood transfusion rate, and (D) readmission rate between ERAS/FTS and conventional care. The size of squares corresponds to the weight of the study in the meta-analysis. The diamond plot represents the overall results of the included trials. The horizontal lines represent the 95\% CI. ERAS, enhanced recovery after surgery; FTS, fast track surgery; 95\% CI, 95\% confidence interval; df, degrees of freedom; IV, inverse variance test; M-H, MantelHaenszel test.

requires a multidisciplinary team, including surgeons, anesthesiologists, and nurses. Each study had a different number of ERAS/FTS elements, leading to inescapable heterogeneity. Finally, some critical outcomes, such as postoperative complications and readmission rates, were not uniformly defined and might have occurred in patients but were not recorded due to follow-up time limitations. Hence, we advise caution in interpreting those results.

\section{Conclusions}

In summary, this is the first systematic review and metaanalysis on ERAS/ FTS in RP. Our results showed that an ERAS/FTS program could shorten LOS, time to first anus exhaust, and reduce the incidence of complications, but could not increase operation time, estimated blood loss, postoperative pain, and had no effect on the rates of blood transfusion and readmission. However, due to the small number of studies available and their underlying heterogeneity, further large, long term, prospective, multicenter follow-up studies and RCTs should be undertaken to confirm our findings.

\section{Acknowledgments}

The authors would like to thank Xu Liu, majoring in Health 
Statistics and Epidemiology in Hebei medical university, for guidance, revision and review of the statistics of this study.

Funding: This work is supported by the following grants: the National Key R\&D Program of China (2017YFC0908004), the National Natural Science Foundation of China (No. 81001137, No. 81874094 and No. 81800590), Hunan Provincial Natural Science Foundation of China (No. 2019JJ40484), Science and Technology Plan Projects of Changsha city (kq1801114), the Project from Health and Family Planning Commission of Hunan Province (No. C20180105) and the Fundamental Research Funds for the Central Universities of Central South University (No. 2019zzts794).

\section{Footnote}

Reporting Checklist: The authors have completed the PRISMA reporting checklist. Available at http://dx.doi. org/10.21037/tau-19-884

Data Sharing Statement: Available at http://dx.doi. org/10.21037/tau-19-884

Conflicts of Interest: All authors have completed the ICMJE uniform disclosure form (available at http://dx.doi. org/10.21037/tau-19-884). The authors have no conflicts of interest to declare.

Ethical Statement: The authors are accountable for all aspects of the work in ensuring that questions related to the accuracy or integrity of any part of the work are appropriately investigated and resolved.

Open Access Statement: This is an Open Access article distributed in accordance with the Creative Commons Attribution-NonCommercial-NoDerivs 4.0 International License (CC BY-NC-ND 4.0), which permits the noncommercial replication and distribution of the article with the strict proviso that no changes or edits are made and the original work is properly cited (including links to both the formal publication through the relevant DOI and the license). See: https://creativecommons.org/licenses/by-nc-nd/4.0/.

\section{References}

1. Bray F, Ferlay J, Soerjomataram I, et al. Global cancer statistics 2018: GLOBOCAN estimates of incidence and mortality worldwide for 36 cancers in 185 countries. CA
Cancer J Clin 2018;68:394-424.

2. Chen FZ, Zhao XK. Prostate cancer: current treatment and prevention strategies. Iran Red Crescent Med J 2013;15:279-84.

3. Ficarra V, Novara G, Artibani W, et al. Retropubic, laparoscopic, and robot-assisted radical prostatectomy: a systematic review and cumulative analysis of comparative studies. Eur Urol 2009;55:1037-63.

4. Tewari A, Sooriakumaran P, Bloch DA, et al. Positive surgical margin and perioperative complication rates of primary surgical treatments for prostate cancer: a systematic review and meta-analysis comparing retropubic, laparoscopic, and robotic prostatectomy. Eur Urol 2012;62:1-15.

5. Nam RK, Cheung P, Herschorn S, et al. Incidence of complications other than urinary incontinence or erectile dysfunction after radical prostatectomy or radiotherapy for prostate cancer: a population-based cohort study. Lancet Oncol 2014;15:223-31.

6. Bardram L, Funch-Jensen P, Jensen P, et al. Recovery after laparoscopic colonic surgery with epidural analgesia, and early oral nutrition and mobilisation. Lancet 1995;345:763-4.

7. Kehlet H. Multimodal approach to control postoperative pathophysiology and rehabilitation. Br J Anaesth 1997;78:606-17.

8. Ni X, Jia D, Guo Y, et al. The efficacy and safety of enhanced recovery after surgery (ERAS) program in laparoscopic digestive system surgery: a meta-analysis of randomized controlled trials. Int J Surg 2019;69:108-15.

9. Gralla $\mathrm{O}$, Haas F, Knoll N, et al. Fast-track surgery in laparoscopic radical prostatectomy: basic principles. World J Urol 2007;25:185-91.

10. Abou-Haidar H, Abourbih S, Braganza D, et al. Enhanced recovery pathway for radical prostatectomy: implementation and evaluation in a universal healthcare system. Can Urol Assoc J 2014;8:418-23.

11. Yaiesh S, Aldousari S, Abul F, et al. Does a standardized algorithm for managing patients post-robotic-assisted radical prostatectomy improve recovery? Experience with the Optimized Surgical Journey. Eur Urol Suppl 2016;15:e1288-9.

12. Nosov AK, Reva SA, Berkut MV, et al. Early recovery program in the radical surgical treatment of patients with prostate cancer: experience of the specialized hospital. Onkourologiya 2016;12:60-9.

13. Huang Z, Yi L, Zhong Z, et al. Comparison of fasttrack versus conventional surgery protocol for patients 
undergoing robot-assisted laparoscopic radical prostatectomy: a Chinese experience. Sci Rep 2018;8:8017.

14. Lin C, Wan F, Lu Y, et al. Enhanced recovery after surgery protocol for prostate cancer patients undergoing laparoscopic radical prostatectomy. J Int Med Res 2019;47:114-21.

15. Parrado CL, Alonso y Gregorio S, Martín-Martínez A, et al. Impact of a clinical pathway in patient care following surgery on laparoscopic radical prostatectomy. Qual Manag Health Care 2008;17:234-41.

16. Sugi M, Matsuda T, Yoshida T, et al. Introduction of an enhanced recovery after surgery protocol for robotassisted laparoscopic radical prostatectomy. Urol Int 2017;99:194-200

17. Graham J, Dickerson S, Longhitano Y, et al. Immediate impact of a multidisciplinary enhanced recovery after surgery program for robotic radical prostatectomies. Clinical Nutrition ESPEN 2019;31:116-7.

18. Magheli A, Knoll N, Lein M, et al. Impact of fast-track postoperative care on intestinal function, pain, and length of hospital stay after laparoscopic radical prostatectomy. J Endourol 2011;25:1143-7.

19. Wan X, Wang W, Liu J, et al. Estimating the sample mean and standard deviation from the sample size, median, range and/or interquartile range. BMC Med Res Methodol 2014; $14: 135$

20. Luo D, Wan X, Liu J, et al. Optimally estimating the sample mean from the sample size, median, mid-range, and/or mid-quartile range. Stat Methods Med Res 2018;27:1785-805.

21. Wolboldt M, Saltzman B, Tenbrink P, et al. Sameday discharge for patients undergoing robot-assisted laparoscopic radical prostatectomy is safe and feasible: results of a pilot study. J Endourol 2016;30:1296-300.

22. Ljungqvist O, Scott M, Fearon KC. Enhanced recovery after surgery: a review. JAMA Surg 2017;152:292-8.

23. Abaza R, Martinez O, Ferroni MC, et al. Same day discharge after robotic radical prostatectomy. J Urol 2019;202:959-63.

24. Cao L, Yang Z, Qi L, et al. Robot-assisted and laparoscopic vs open radical prostatectomy in clinically localized prostate cancer: perioperative, functional, and oncological outcomes: a systematic review and meta-analysis. Medicine (Baltimore) 2019;98:e15770.

25. Pushan Z, Manbiao C, Sulai L, et al. The impact of perioperative blood transfusion on survival and recurrence after radical prostatectomy for prostate cancer: a systematic review and meta-analysis. J Cancer Res Ther
2018;14:S701-7.

26. Master VA. Leaning in to understand hospital readmission. J Urol 2014;192:10-12.

27. Holte K, Nielsen KG, Madsen JL, et al. Physiologic effects of bowel preparation. Dis Colon Rectum 2004;47:1397-402.

28. Nygren J, Thacker J, Carli F, et al. Guidelines for perioperative care in elective rectal/pelvic surgery: Enhanced Recovery After Surgery (ERAS(®)) Society recommendations. World J Surg 2013;37:285-305.

29. Giglio MT, Marucci M, Testini M, et al. Goal-directed haemodynamic therapy and gastrointestinal complications in major surgery: a meta-analysis of randomized controlled trials. Br J Anaesth 2009;103:637-46.

30. Moraca RJ, Sheldon DG, Thirlby RC. The role of epidural anesthesia and analgesia in surgical practice. Ann Surg 2003;238:663-73.

31. Dougherty SH, Simmons RL. The biology and practice of surgical drains. Part II. Curr Probl Surg 1992;29:633-730.

32. Niesel T, Partin AW, Walsh PC. Anatomic approach for placement of surgical drains after radical retropubic prostatectomy: long-term effects on postoperative pain. Urology 1996;48:91-4.

33. Noldus J, Huland H. Early complications of radical prostatectomy. Pelvic vein compression caused by wound drainage. Urologe A 1994;33:172-4.

34. Musser JE, Assel M, Guglielmetti GB, et al. Impact of routine use of surgical drains on incidence of complications with robot-assisted radical prostatectomy. Journal of endourology 2014;28:1333-7.

35. Sachedina N, De Los Santos R, Manoharan M, et al. Total prostatectomy and lymph node dissection may be done safely without pelvic drainage: an extended experience of over 600 cases. Can J Urol 2009;16:4721-5.

36. Sharma S, Kim HL, Mohler JL. Routine pelvic drainage not required after open or robotic radical prostatectomy. Urology 2007;69:330-3.

37. Canes D, Cohen MS, Tuerk IA. Laparoscopic radical prostatectomy: omitting a pelvic drain. Int Braz J Urol 2008;34:151-8.

38. Schatzl G, Madersbacher S, Hofbauer J, et al. The impact of urinary extravasation after radical retropubic prostatectomy on urinary incontinence and anastomotic strictures. Eur Urol 1999;36:187-90.

39. Reuvers S, Zonneveld W, Meiland-van Bakel M, et al. Indwelling urinary catheter-related problems after laparoscopic radical prostatectomy. J Wound Ostomy Continence Nurs 2016;43:420-4

40. Lista G, Lughezzani G, Buffi NM, et al. Early catheter 
removal after robot-assisted radical prostatectomy: results from a prospective single-institutional randomized trial (Ripreca Study). Eur Urol Focus 2020;6:259-66.

41. Brassetti A, Proietti F, Cardi A, et al. Removing the urinary catheter on post-operative day 2 after robotassisted laparoscopic radical prostatectomy: a feasibility study from a single high-volume referral centre. J Robot Surg 2018;12:467-73.

42. Gratzke C, Dovey Z, Novara G, et al. Early Catheter removal after robot-assisted radical prostatectomy: surgical

Cite this article as: Lv Z, Cai Y, Jiang H, Yang C, Tang C, Xu H, Li Z, Fan B, Li Y. Impact of enhanced recovery after surgery or fast track surgery pathways in minimally invasive radical prostatectomy: a systematic review and meta-analysis. Transl Androl Urol 2020;9(3):1037-1052. doi:10.21037/tau-19884 technique and outcomes for the Aalst technique (ECaRemA Study). Eur Urol 2016;69:917-23.

43. Grass F, Schäfer M, Demartines N, et al. Normal diet within two postoperative days-realistic or too ambitious? Nutrients 2017;9:1336.

44. Bundgaard-Nielsen M, Jørgensen CC, Jørgensen TB, et al. Orthostatic intolerance and the cardiovascular response to early postoperative mobilization. Br J Anaesth 2009;102:756-62. 